\title{
Tests of Perturbative QCD and Jet Physics
}

\author{
J. Womersley \\ Fermi National Accelerator Laboratory, \\ Batavia, Illinois 60510
}

Quantum Chromodynamics (QCD) is a vast domain. In the past twelve months, no less than 473 papers with "QCD" in the title were submitted to the hep-ph archive (admittedly, this includes such titles as "Cosmological QCD Phase Transition and Dark Matter"). More relevant, perhaps, is to note that 93 QCD-related abstracts were submitted to the 1999 Europhysics Conference on High Energy Physics (EPS99). I shall therefore have to be selective, and will organize this presentation by final state: jets, photons, weak bosons and heavy flavor.

I would like to thank everyone who helped me put this presentation together, and extend my apologies to all those whose work had to be omitted or brutally summarized. I also ask the audience's indulgence for any biases from my particular background as a Tevatron experimenter.

\section{Jets}

\subsection{Inclusive Jet Cross Sections at $\sqrt{s}=1.8 \mathrm{TeV}$}

So much has been said about the high- $E_{T}$ behavior of the inclusive jet cross section at the Tevatron that it is difficult to know what can usefully be added (see Fig. 1). The measured central inclusive jet cross sections, from CDF [1] and DO [2], compared with the NLO theory, are shown in Fig. 2 (note that the CDF figure does not include systematic errors). The impression one gets is that there is a marked excess above QCD in the CDF data, which is not observed at DO.

In order to compare with CDF, DO carried out an analysis in exactly the same rapidity interval $(0.1<|\eta|<0.7)$. The results [3] are shown in Fig. 3. Firstly we note that there is no actual discrepancy between the datasets. Secondly, for this plot the theoretical prediction was made using the CTEQ4HJ parton distribution, which has been adjusted to give an increased gluon density at large $x$ while not violating any experimental constraints (except perhaps fixed target photon production data, which in any case require big corrections before they can be compared to QCD, as we shall see later). The result of this increased gluon content is improved agreement especially with the CDF data points.

What then have we learned from this issue? In my opinion, whether the CDF data show a real excess above QCD, or just a "visual excess," depends critically 


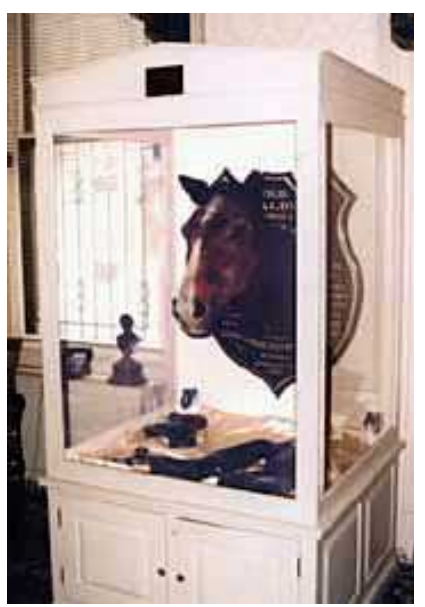

Figure 1: "The horse is dead."

on understanding the systematic errors and their correlations as a function of $E_{T}$. Whether nature has actually exploited the freedom to enhance gluon distributions at large $x$ will only be clear with the addition of more data - the factor of 20 increase in luminosity in Run II will extend the reach significantly in $E_{T}$ and should make the asymptotic behavior clearer. Whatever the Run II data show, this has been a useful lesson; it has reminded us all that parton distributions have uncertainties, whether made explicit or not, and that a full understanding of experimental systematics and their correlations is needed to understand whether experiments and theory agree or disagree.

DO [4] have extended their measurement of inclusive jet cross sections into the forward region. Figure 1 shows the measured cross sections up to $|\eta|=3$. They are in good agreement with NLO QCD over the whole range of pseudorapidity and transverse energy.

\subsection{Dijet Production}

Both Tevatron experiments have also studied dijet final states. CDF [5] has presented cross sections for processes with one central jet $\left(0.1<\left|\eta_{1}\right|<0.7\right)$ and one jet allowed forward ( $\left|\eta_{2}\right|$ up to 3.0). In Fig. 5 these are compared with the NLO QCD prediction ast a function of the central jet's transverse energy $\left(E_{T 1}\right)$. The data show an excess above the theory for large $E_{T 1}$, just as seen in the inclusive cross section; but since these events are common to both samples, this is not surprising.

DO have measured [4] the cross sections for dijet production with both same-side $\left(\eta_{1} \approx \eta_{2}\right)$ and opposite-side $\left(\eta_{1} \approx-\eta_{2}\right)$ topologies, for four bins of $|\eta|$ up to 2.0. The 

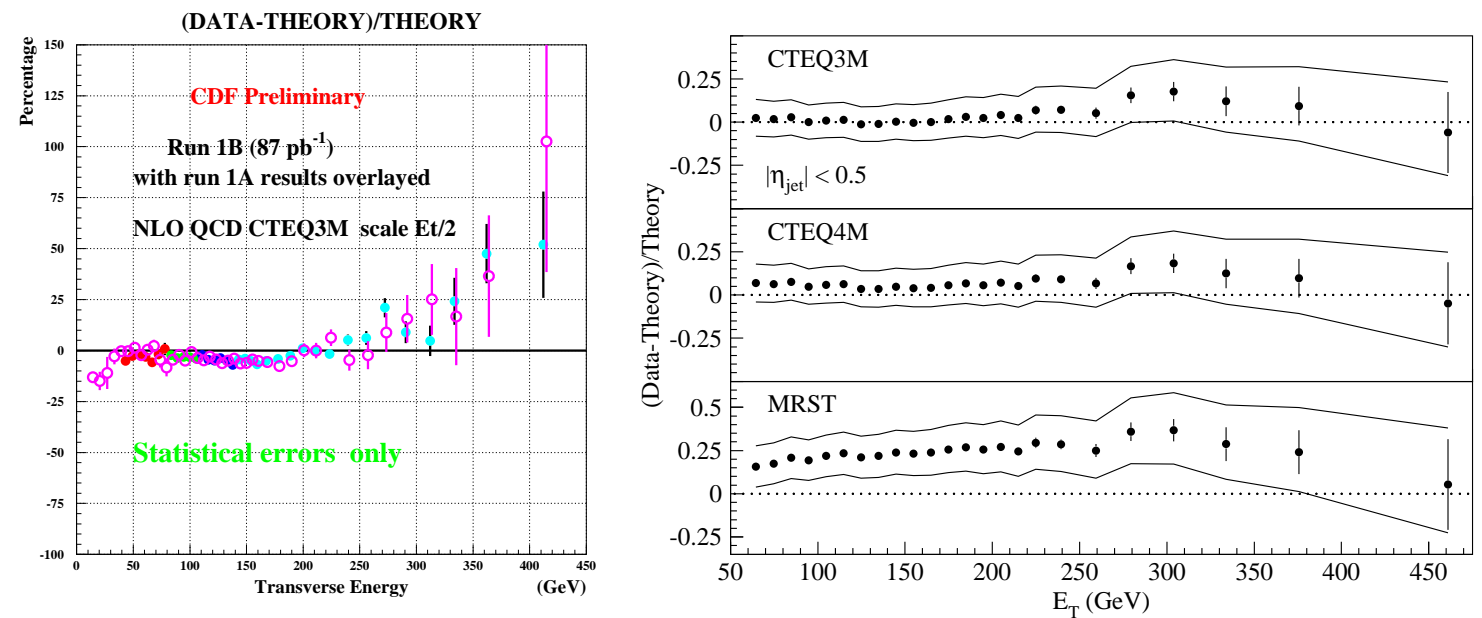

Figure 2: Inclusive jet cross sections measured at the Tevatron by CDF[1] (left, for $0.1<|\eta|<0.7$ ) and DO [2] (right, for $|\eta|<0.5$ ), all normalized to the NLO QCD prediction.

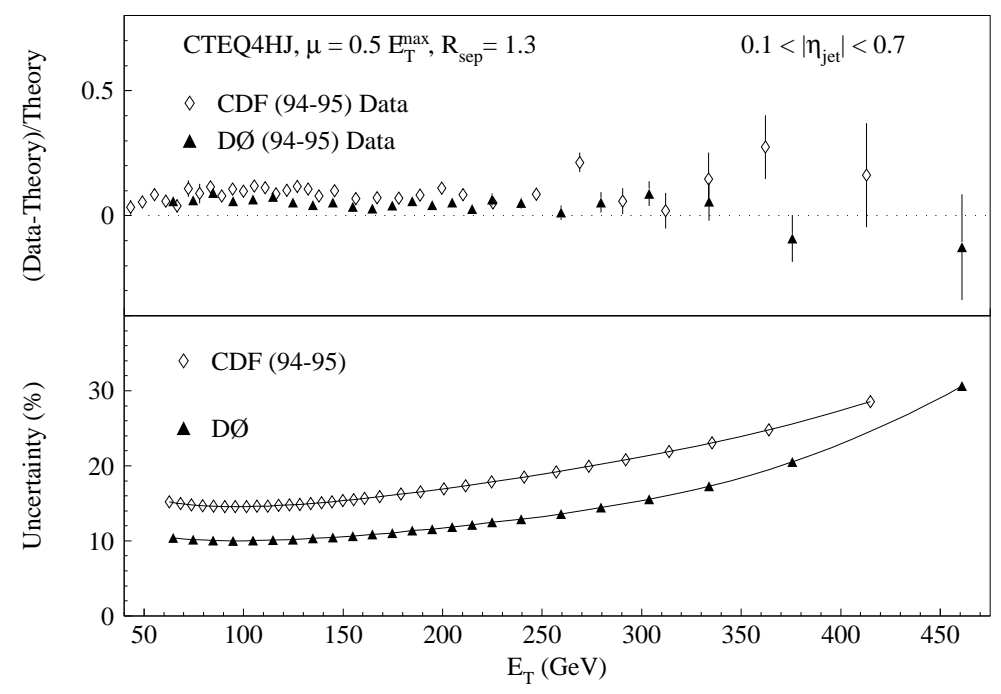

Figure 3: Inclusive jet cross sections for $0.1<|\eta|<0.7$ from CDF and DO, compared with CTEQ4HJ distribution; and size of the systematic errors on the two measurements. Taken from [3]. 

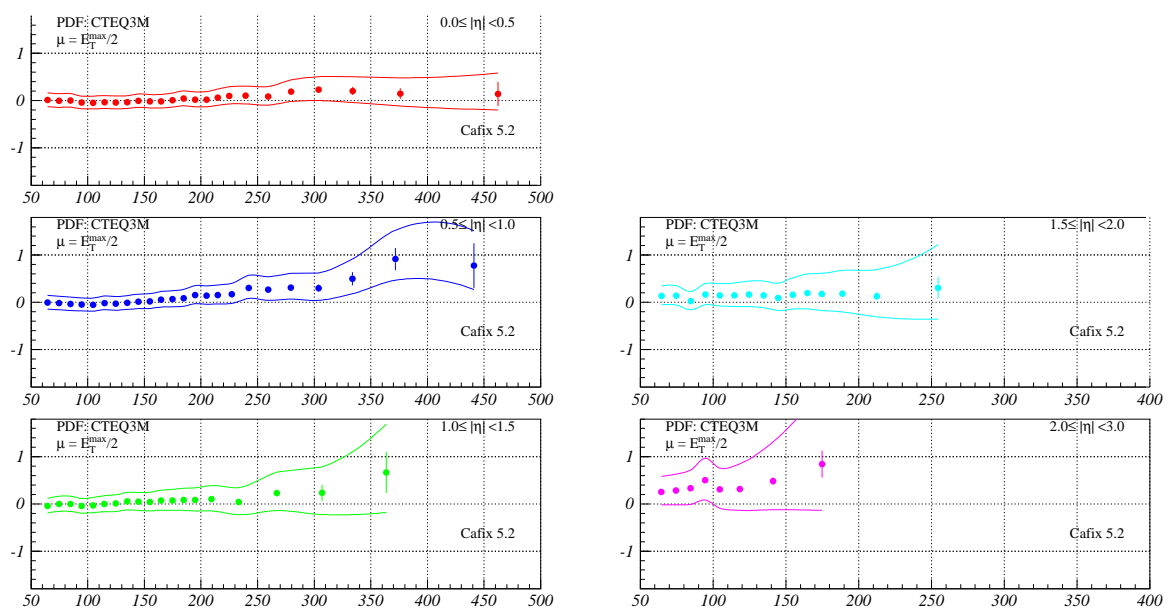

Figure 4: Inclusive jet cross sections measured up to $|\eta|=3$ by DO [4], compared to the NLO QCD prediction (from JETRAD using the CTEQ3M parton distributions).

results are all in good agreement with the NLO QCD prediction, as seen in Fig. 6.

\subsection{Jet Cross Sections at $\sqrt{s}=630 \mathrm{GeV}$}

Both CDF [6] and DO[7] have measured the ratio of jet cross sections at $\sqrt{s}=1800$ and $630 \mathrm{GeV}$, exploiting a short period of data taking at the latter center of mass energy at the end of Run I. This ratio is expected to be a rather straightforward quantity to measure and to calculate. The ratio as a function of scaled jet transverse energy $x_{T}=2 E_{T} / \sqrt{s}$ is shown in Fig.77. Unfortunately the two experiments are not obviously consistent with each other (especially at low $x_{T}$ ) or with NLO QCD (at any $x_{T}$ ). At least two explanations have been suggested for the discrepancy. Firstly, different renormalization scales could be used for the theoretical calculations at the two energies. While allowed, this seems unappealing. Glover has suggested that such a procedure is in fact natural when a scaling variable like $x_{T}$ is used; because $x_{T}$ differs by a factor of about three between the two center of mass energies for a given $E_{T}$, a factor of three difference in the renormalization scales is appropriate. An alternative explanation is offered by Mangano, who notes that a shift in jet energies by of order $3 \mathrm{GeV}$ which might arise from non-perturbative effects would bring the data in line with the prediction. Such effects might include losses outside the jet cone, underlying event energy, and intrinsic transverse momentum of the incoming partons; one might be under or over-correcting the data and the two experiments might even obtain different results depending on how the corrections were done (based on data or Monte 

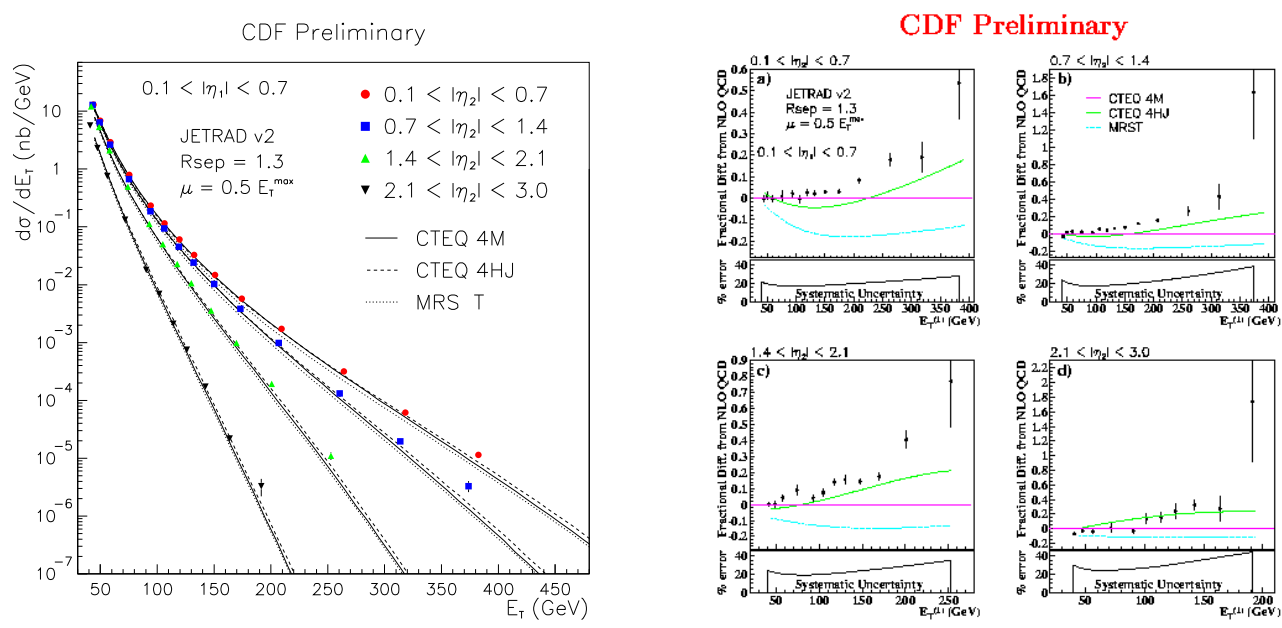

Figure 5: Dijet cross sections measured by CDF[5] for events with one central jet $0.1<\left|\eta_{1}\right|<0.7$ and one jet allowed forward; left, as a function of the central jet $E_{T}$ for various bins of $\left|\eta_{2}\right|$, and right, normalized to the NLO QCD prediction (from JETRAD).

Carlo, for example). It seems that more work, both theoretical and exerimental, is needed before this question can be resolved.

\subsection{BFKL}

DO 8] have used the $630 \mathrm{GeV}$ data to make a measurement which a cynic might perhaps describe as "yet another attempt to find an observable which displays BFKL behaviour." The ratio of cross sections at 1800 and $630 \mathrm{GeV}$ is measured for dijets with large rapidity separations, using bins such that $x$ and $Q^{2}$ are the same in the two datasets (and therefore the parton distributions cancel, to first order). Figure 8 shows this ratio as a function of the rapidity separation $\Delta \eta$ at $630 \mathrm{GeV}$. The ratio is much larger than unity, and rises with rapidity separation, qualitatively like the BFKL expectation - but also like HERWIG. Maybe we are indeed seeing BFKL dynamics, but given that we apparently can't predict the ratio of inclusive cross sections between the two energies, I would caution against inferring too much.

\subsection{Jet Structure at the Tevatron}

All the results presented so far have used a cone jet finder. By running a $k_{T}$ jet finder inside previously identified jets, one can count the number of "subjets" or energy 

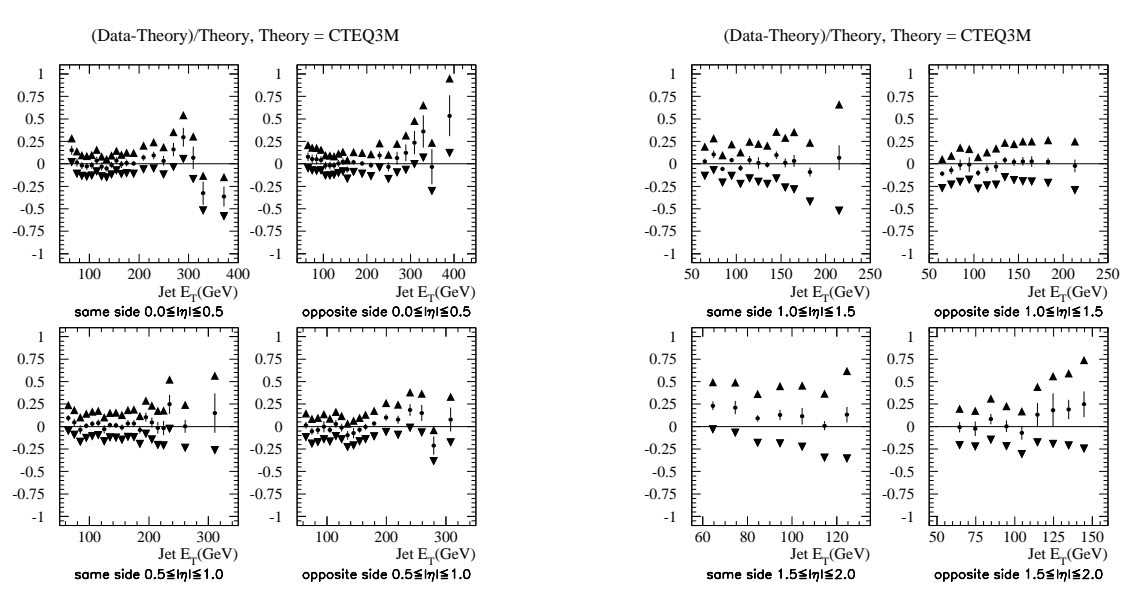

Figure 6: Dijet jet cross sections measured up to $|\eta|=2$ by DO [4], for same side and opposite side topologies, compared to the NLO QCD prediction (from JETRAD using the CTEQ3M parton distributions).

clusters. This allows the perturbative part of fragmentation to be studied. DO 9 have made such a measurement and, by comparing jets of the same $E_{T}$ and $\eta$ at $\sqrt{s}=1800$ and $630 \mathrm{GeV}$, have inferred the composition of quark and gluon jets. The extracted subjet multiplicity $M$ for the two species is shown in Fig.9. The ratio of $M-1$ for the two cases, which might naively be expected to equal the ratio of gluon and quark colour charges, is found to be $1.91 \pm 0.04$, compared with $1.86 \pm 0.04$ from HERWIG.

These last three measurements all relied on a very short period of Tevatron running at $630 \mathrm{GeV}$, which was surprisingly productive. Though it is politically difficult to get reduced energy running time when one is searching for new physics, I suspect we may want to do something like this once again in the next run.

\subsection{Jet Production at HERA}

Many studies of jet production have also been carried out at HERA, by H1 and ZEUS 10. Inclusive jet, dijet and three-jet cross sections have been measured as a function of jet $E_{T}$, pseudorapidity and $Q^{2}$, typically using a $k_{T}$ jet finder. The results are in good agreement with NLO (where available) or LO QCD. ZEUS have also reported a study of subjets similar to that described above 11]; they find that the subjet multiplicity rises as the jet becomes more forward, which is consistent with the expectation that more gluon jets would be produced in this region of phase space (and also consistent with the HERWIG Monte Carlo). 


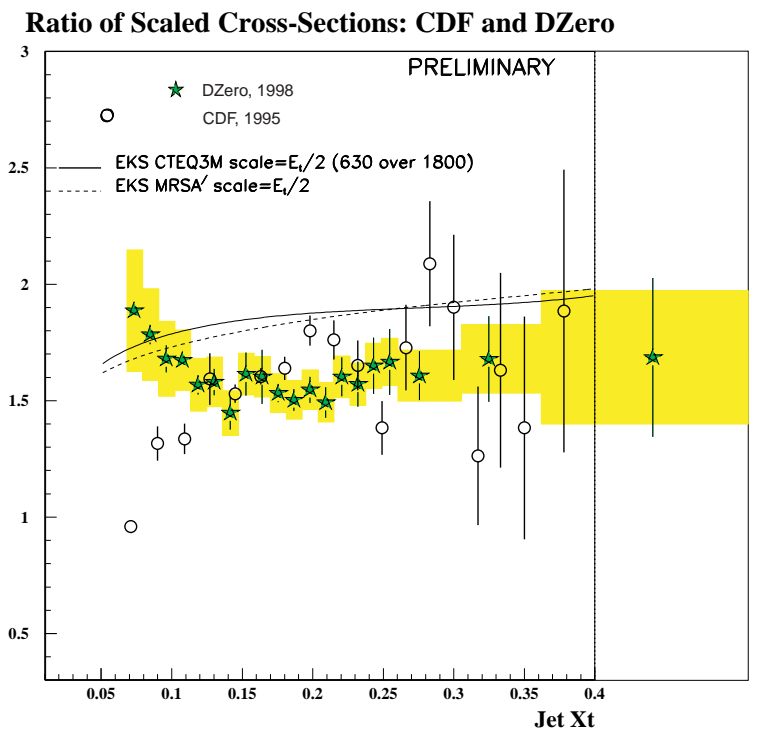

Figure 7: Ratio of jet cross sections at $\sqrt{s}=1800 \mathrm{GeV}$ to $\sqrt{s}=630 \mathrm{GeV}$, as a function of $x_{T}=2 E_{T} / \sqrt{s}$, as measured by $\mathrm{CDF}[6]$ and $\mathrm{DO}[7]$ and as predicted by NLO QCD

\section{DØ Preliminary}

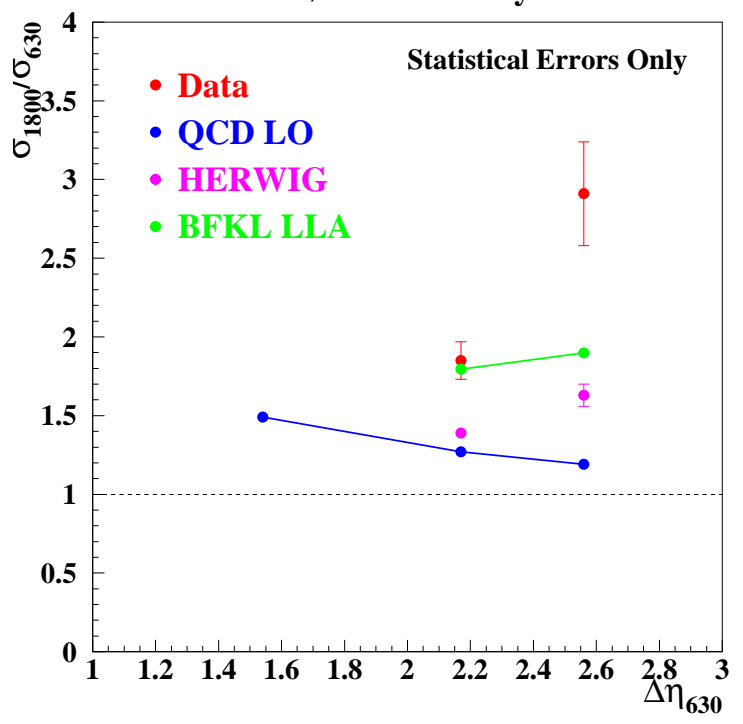

Figure 8: Ratio of cross section at $\sqrt{s}=1800 \mathrm{GeV}$ to that at $\sqrt{s}=630 \mathrm{GeV}$, for events with dijets widely separated in rapidity 8$]$. 


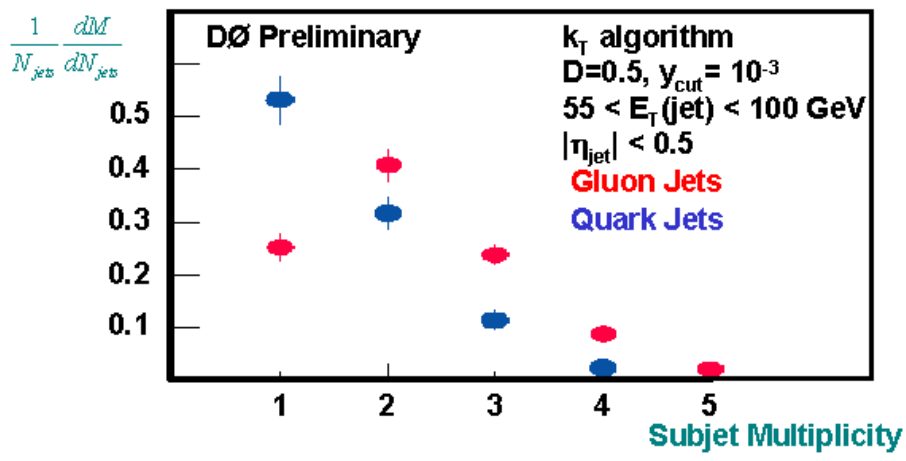

Figure 9: Subjet multiplicities measured by DO using a $k_{T}$ algorithm to find clusters within jets; distributions for quark and gluon jets are inferred using $\sqrt{s}=1800 \mathrm{GeV}$ and $\sqrt{s}=630 \mathrm{GeV}$ data [9].

\section{Direct Photon Production}

Historically, many authors hoped that measurements of direct (or prompt) photons would provide a clean test of QCD, free from the systematic errors associated with jets, and would help pin down parton distributions. In fact photons have not lived up to this promise - instead they revealed that there may be unaccounted-for effects in QCD cross sections at low $E_{T}$. (Because photons can typically be measured at lower energies than jets, they provide a way of exploring the low- $E_{T}$ regime). Results from the Tevatron experiments [12] [13] [14] are shown in Fig. 10. While the general agreement with the NLO calculation of Owens and collaborators [15] is good, there is a definite tendency for the data to rise above the theory at low energies.

An often-invoked explanation for this effect is that there is extra transverse momentum smearing of the partonic system due to soft gluon radiation. The magnitude of the smearing, or " $k_{T}$ ", is typically a few GeV (at the Tevatron), motivated in part by the experimentally measured $p_{T}$ of the $\gamma \gamma$ system in diphoton production which peaks around $3 \mathrm{GeV}[16]$. Inclusion of such $k_{T}$ as a Gaussian smearing in the calculation gives much better agreement with the data, as shown in Fig. 11.

One should note that a shape a bit closer to that observed in the data can be obtained in other ways. Vogelsang et al. [17 use a NLO treatment of fragmentation and allow the renormalization and factorization scales to differ, yielding the curves shown in Fig.12 without any $k_{T}$.

Much larger deviations from QCD are observed in fixed-target experiments such as E706 at Fermilab [18]. Again, Gaussian smearing (with $k_{T} \approx 1.2 \mathrm{GeV}$ in this case) can account for the data, as shown in Fig. 13. A rather different view is expressed by 

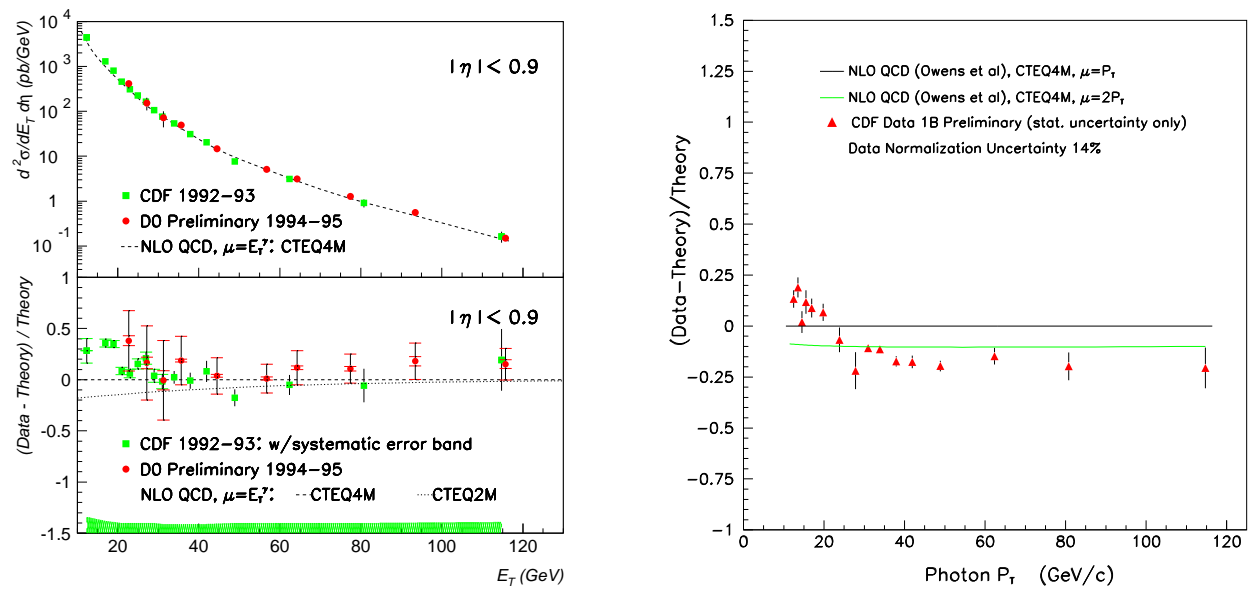

Figure 10: Inclusive isolated direct photon cross sections at the Tevatron; the left hand plot shows CDF 12$]$ and DO[13] measurements and the right hand plot shows the latest CDF results 14 (statistical errors only). All are compared with the NLO QCD prediction of Owens et al. [15].
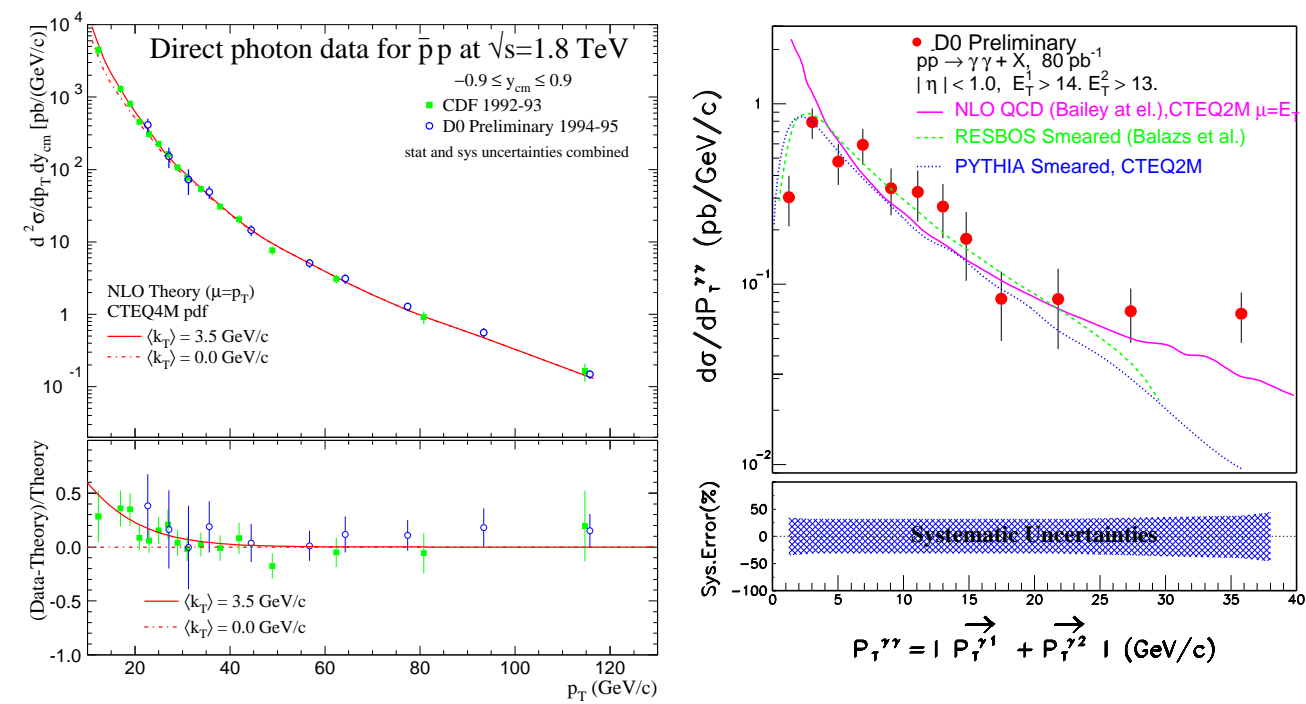

Figure 11: The isolated photon cross section at the Tevatron (left hand plots) agrees rather better with QCD if $3.5 \mathrm{GeV}$ of transverse momentum smearing (" $k_{T}$ ") is added to account for soft gluon emission. The magnitude of the $k_{T}$ smearing is consistent with the most probable momentum of $\gamma \gamma$ pairs (right hand plot) [16. 


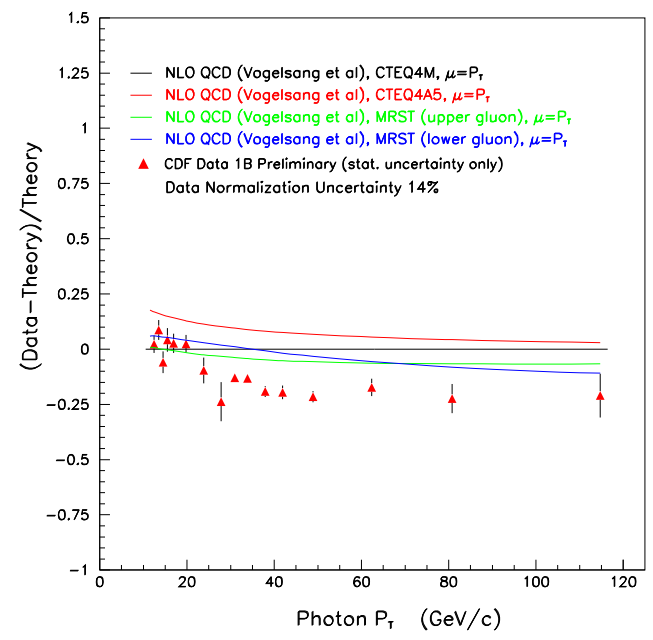

Figure 12: Latest CDF isolated photon cross section normalized to the NLO QCD prediction of Vogelsang et al. [17], showing various choices of PDF and renormalization scale within this prediction. Statistical errors only.

Aurenche and collaborators [19], who find their calculations, sans $k_{T}$, to be consistent with all data with the sole exception of E706 (see Fig. 14). They say "it does not appear very instructive to hide this problem by introducing an arbitrary parameter fitted to the data at each energy." Indeed, I believe that Gaussian smearing has told us pretty much all that it can. Its predictivce power is small: what happens to forward photons, for example? The "right way" to treat soft gluon emission should be through a resummation calculation which works nicely for $\gamma \gamma$ and $W / Z$ transverse momentum distributions. Unfortunately, this approach does not seem to model the E706 data, which still lie a factor of two or more above the resummed QCD calculations 20 21 (Fig. 15). I do not know if there are other terms that can be resummed, of whether this should be taken as an implication that the whole idea of soft gluons being responsible for this discrepancy is mistaken.

As an interesting aside, we may note that the ZEUS measurement of prompt photon production at HERA[22], which covers a similar range of transverse momenta to E706, agrees well with NLO QCD without any need for $k_{T}$ (Fig. 16). It is true that the agreement is only good if the "resolved" photon contribution to the DIS process is included, and this does form a kind of resummation. It has also been pointed out that the enhancements from $k_{T}$ would be smaller here than for E706 as the cross section is less steeply falling, with only a $20 \%$ enhancement expected at $E_{T}=5 \mathrm{GeV}$.

In summary, direct photon production has proved extremely interesting and remains quite controversial. The appropriateness of a Gaussian $k_{T}$ treatment is still 

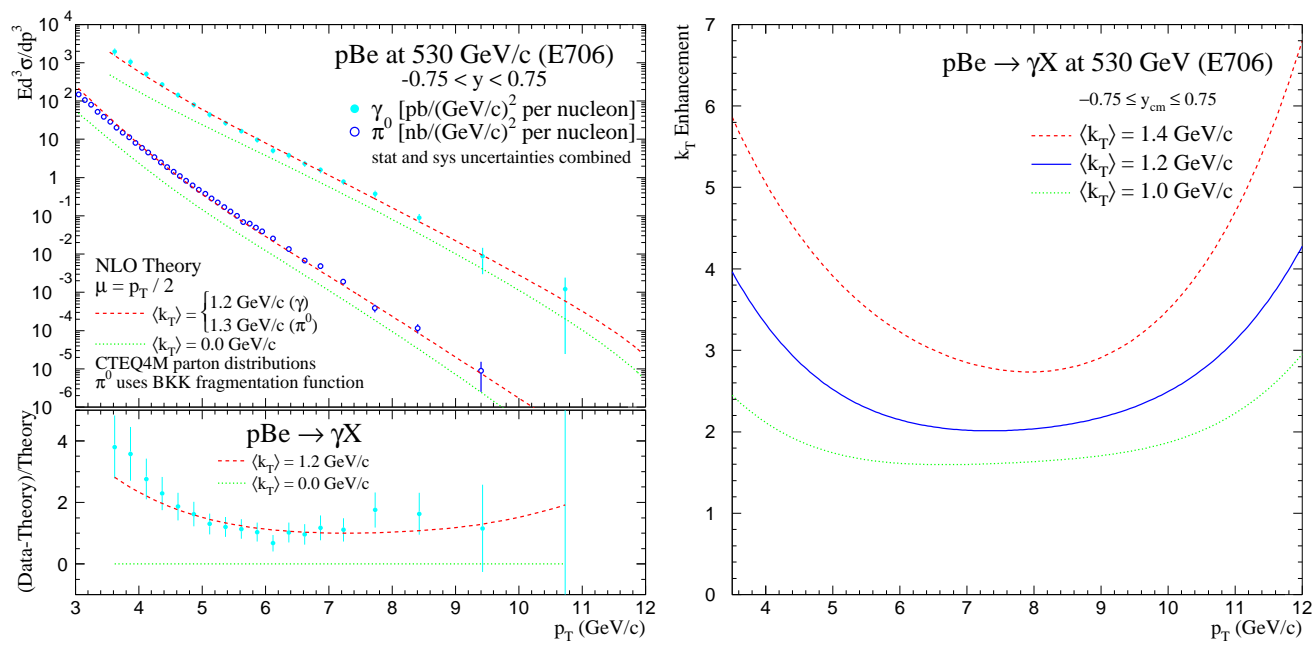

Figure 13: The left hand plot shows the isolated photon and $\pi^{0}$ cross sections measured by E706 18], compared with the NLO QCD prediction with and without additional $k_{T}$ smearing. The right hand plot shows the increase in the cross section obtained when various values of $k_{T}$ are used.

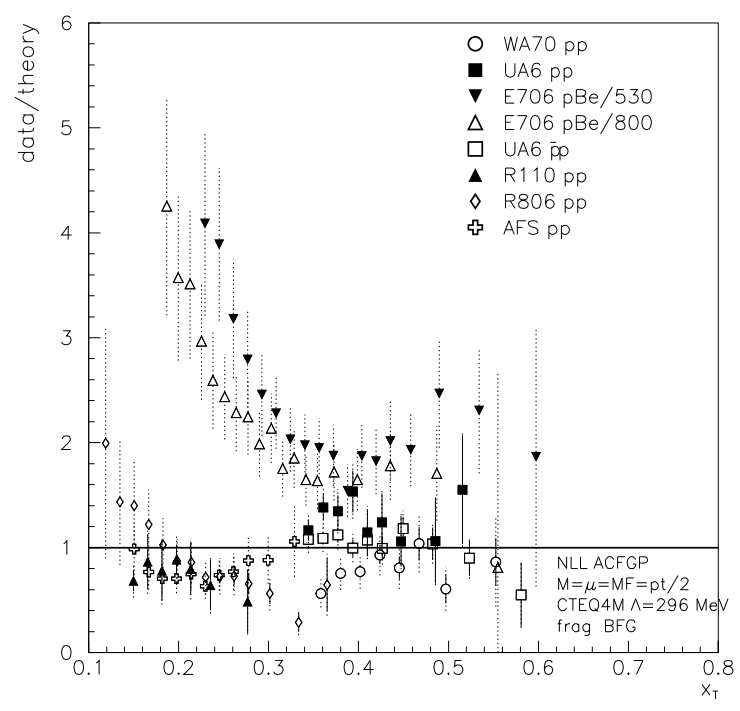

Figure 14: Compilation of isolated photon cross sections compared with NLO QCD, as a function of $x_{T}=2 E_{T} / \sqrt{s}$. From Aurenche et al. [19] 

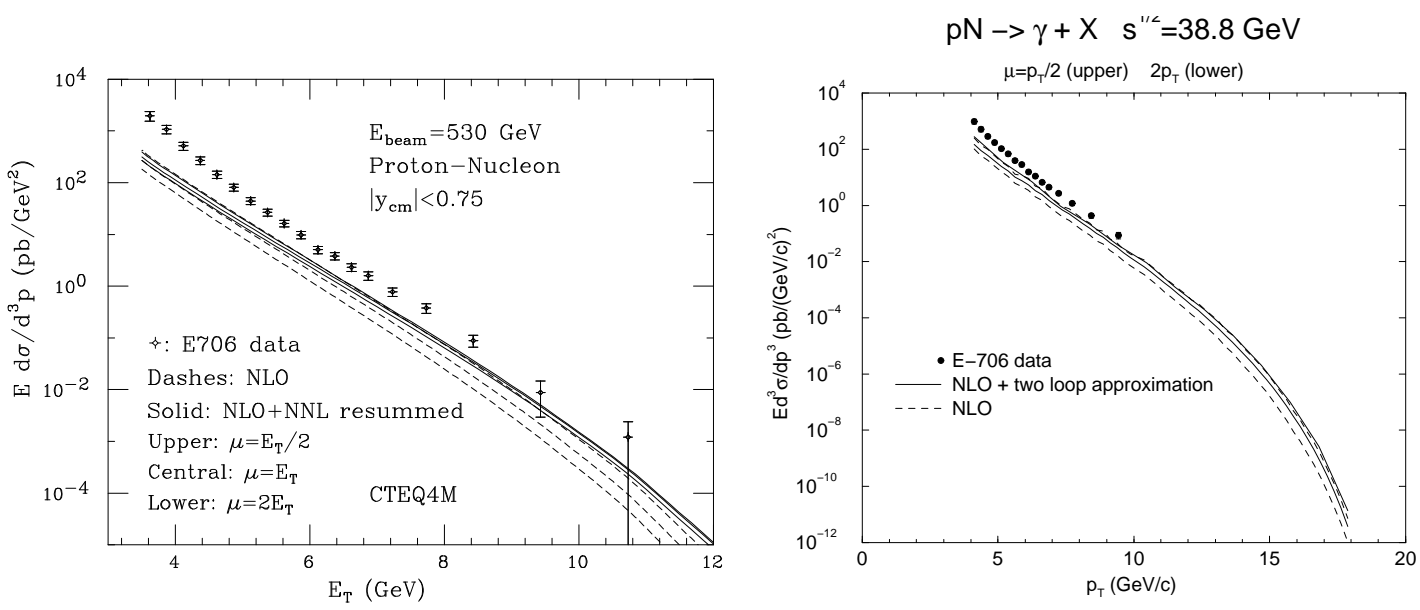

Figure 15: Resummed calculations of isolated photon production compared with the E706 data; left, by Catani et al. [20] and right, by Kidonakis [21].

hotly debated, the experiments may not all be consistent, and resummation has not proved to be the answer.

\section{Weak Bosons}

\subsection{Z Transverse Momentum}

DO has new results on the transverse momentum distribution of the $Z$ boson 223. Figure 17 shows the data compared with a variety of QCD predictions. Clearly the fixed-order NLO QCD is not a good match for the data, while the resummed formalism of Ladinsky and Yuan 24] fits rather well. On the other hand the resummed calculations of Davies, Webber and Stirling[25], and of Ellis and Veseli[26], do not offer quite as good a description of the data.

\section{$3.2 W+$ jets}

DO used to show a cross section ratio $(W+1$ jet $) /(W+0$ jet $)$ which was badly in disagreement with QCD. This is no longer shown: the data were basically correct, but there was a bug in the way DO extracted rhe ratio from the DYRAD theory calculation.

Recent CDF measurements of the $W+$ jets cross sections 27] agree well with QCD, as shown in Fig. 18. 


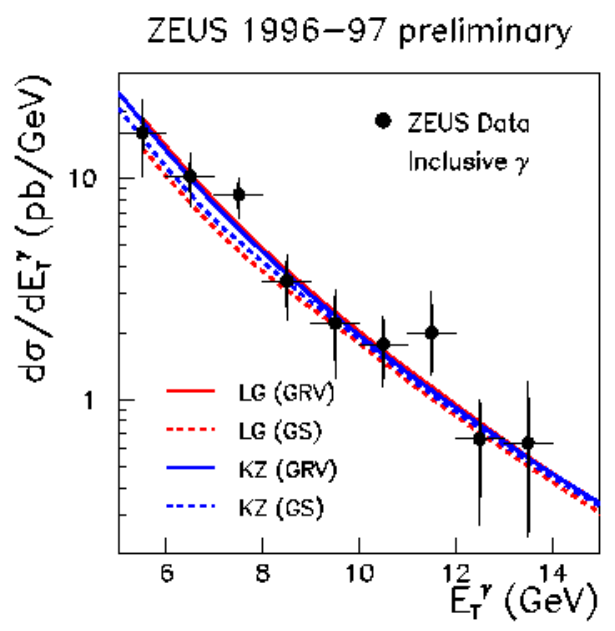

Figure 16: Isolated photon cross section as measured by ZEUS, compared to the NLO QCD prediction 22].

\section{Heavy Flavour Production}

At the Tevatron, the measured inclusive $b$ and $B$-meson production cross sections continue to lie a factor of about two above the NLO QCD expectation. This is seen by both CDF 28 and DO 29] in the central and forward regions (the difference is perhaps even larger for forward $b$ production, as seen in Fig. 19). On the other hand, QCD does a good job of predicting the shape of inclusive distributions, and of the correlations between $b$ quark pairs, so it seems unlikely that any exotic new production mechanism is responsible for the higher than expected cross section.

There are now results on heavy quark production at LEP 2. In particular, L3 has reported [30] the first observation of $b$ production in $\gamma \gamma$ collisions $\left(e^{+} e^{-} \rightarrow e^{+} e^{-} b \bar{b}\right)$. As shown in Fig. 20 the cross section is, once again, 2-3 times the QCD expectation. The picture is rather similar at HERA. H1[31] and ZEUS[32] have reported $b$ production cross sections in $\gamma p$ collisions, using leptons to tag $b$-jets. As shown in Fig. 21 the cross section is at least a factor of two above the QCD prediction. We therefore have a picture of consistent experimental results which are unfortunately all inconsistent with theory!

If the heavy flavour is heavy enough, QCD seems to work rather better. The current state of measured and predicted top cross sections is summarised in Table 1. This includes the latest (revised downward) CDF measurement. There is an excellent agreement between data and theory. 

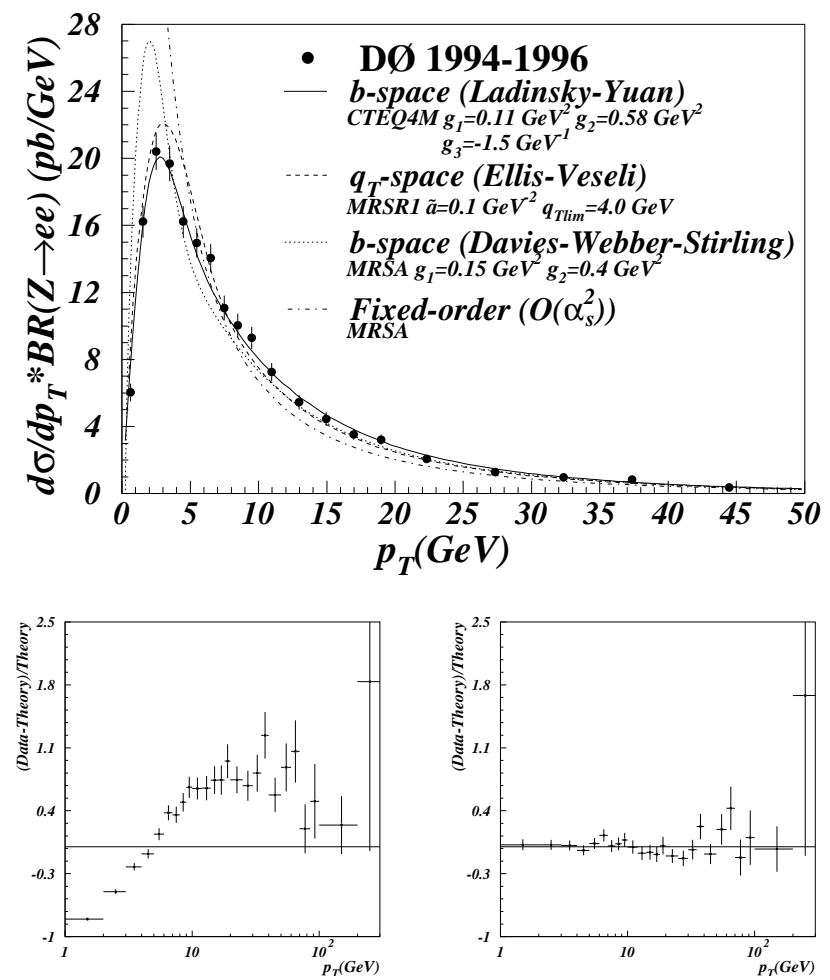

Figure 17: Transverse momentum distribution of the $Z$, as measured by DO 23. The upper plot shows the data and various calculations. The lower left shows the data normalized to the fixed-order QCD prediction and the lower right shows the data normalized to the resummed calculation of Ladinsky and Yuan 24.

\section{Measurements of $\alpha_{s}$}

The strong coupling $\alpha_{s}$ is a fundamental parameter of QCD. Its value cannot be calculated, but must be determined experimentally. A number of new measurements have been reported recently.

Deep inelastic scattering data is now being interpreted in an NNLO framework. Santiago and Ynduráin report [38] an extraction of $\alpha_{s}$ from $F_{2}$ measured at SLAC, BCDMS, E665 and HERA; they obtain $\alpha_{s}\left(m_{Z}\right)=0.1163 \pm 0.0023$. Kataev, Parente and Sidorov 39] extracted $\alpha_{s}$ from $x F_{3}$ measured at CCFR and obtain $\alpha_{s}\left(m_{Z}\right)=$ $0.118 \pm 0.006$.

The LEP electroweak working group has reported 40] new values from LEP 1/SLD $Z$ pole data. The full Standard Model fit yields a value of $\alpha_{s}\left(m_{Z}\right)=0.119 \pm 0.003$ 

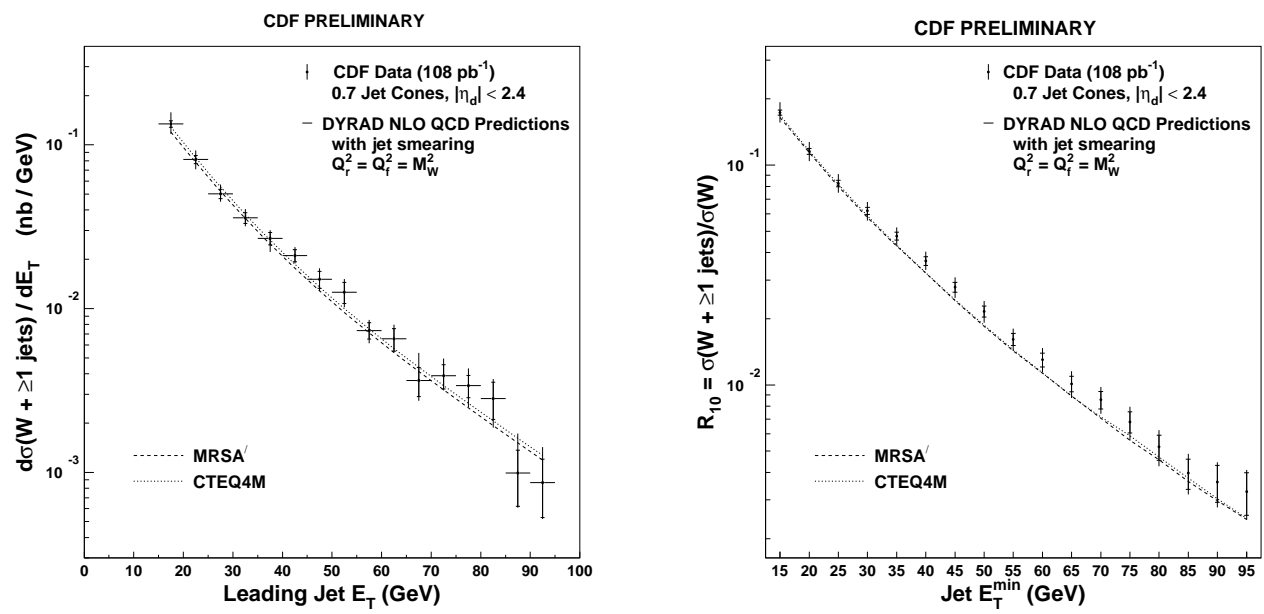

Figure 18: The left hand plot shows the inclusive cross section for $W+$ jets production at the Tevatron, as measured by CDF 27]. The right hand plot shows the ratio of the $W+$ jets cross section to the inclusive $W$ cross section.

while the ratio of the $Z$ partial widths to hadrons and leptons gives $\alpha_{s}\left(m_{Z}\right)=0.119 \pm$ $0.004_{-0}^{+0.003}\left(m_{H}\right)$. The LEP experiments have all extracted $\alpha_{s}$ from event shapes, charged particle and jet multiplicities at $\sqrt{s}=130-196 \mathrm{GeV}$. Monte Carlo programs are used to model non-perturbative effects. Both L3 41] and OPAL[42] have nicely demonstrated the running of $\alpha_{s}$; in the case of L3, by using radiative events to access a lower $\sqrt{s}$, and in OPAL's case by combining their data with that of JADE. Typical uncertainties on $\alpha_{s}\left(m_{Z}\right)$ from the LEP2 data are around \pm 0.006 .

At HERA, $\alpha_{s}$ has been extracted from the inclusive jet rate and the dijet rate $(\mathrm{H} 1433)$ and the dijet fraction (ZEUS[44]). The values of $\alpha_{s}\left(m_{Z}\right)$ obtained are consistent with the world average, with uncertainties of around $\pm 0.005-0.008$.

\begin{tabular}{|l|c|}
\hline Authors & Cross Section $(\mathrm{pb})$ \\
\hline \hline CDF[33] & $6.5_{-1.4}^{+1.7}\left(\right.$ at $\left.m_{t}=175 \mathrm{GeV}\right)$ \\
DO[34] & $5.9 \pm 1.7\left(\right.$ at $\left.m_{t}=172 \mathrm{GeV}\right)$ \\
\hline Bonciani et al. [35] & $5.0 \pm 1.6$ \\
Berger and Contopanagos[36] & $5.6_{-0.4}^{+0.1}$ \\
Kidonakis 37] & 7.0 \\
\hline
\end{tabular}

Table 1: Top production cross sections at the Tevatron, measured and predicted. 

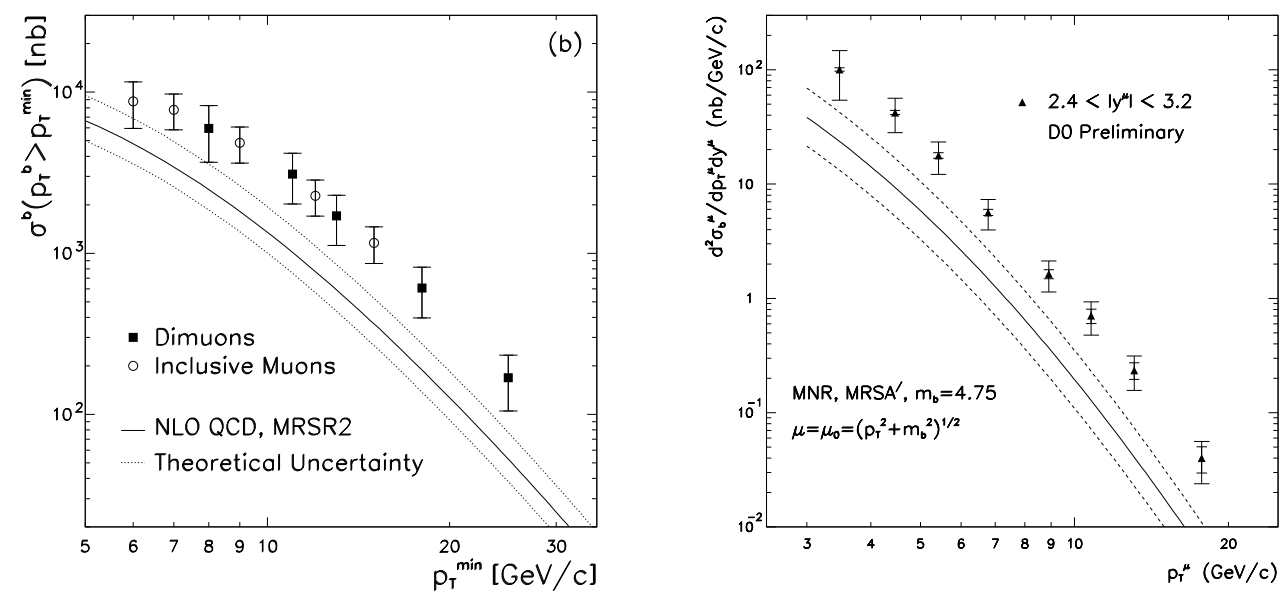

Figure 19: Cross sections for $b$ production at the Tevatron compared with NLO QCD predictions, as measured by DO [29]; left, central rapidity region, and right, forward.

S. Bethke[45] has kindly provided me with an updated world average value of $\alpha_{s}\left(m_{Z}\right)$ for this meeting. It is based on 25 measurements listed in Fig. 22:

$$
\alpha_{s}\left(m_{Z}\right)=0.119 \pm 0.004
$$

If one uses only complete NNLO QCD results (the filled symbols in Fig.22) one obtains:

$$
\alpha_{s}\left(m_{Z}\right)=0.119 \pm 0.003 .
$$

We note excellent consistency bwteeen low and high energy data, and between deep inelastic scattering, electron-positron and hadron collider data. Changes from the previous world average 46 are minimal.

\subsection{Consistency Tests}

At this point we know the value of $\alpha_{s}$ rather well - it is hard to point to a prediction which is limited by its precision. Hence some of the more interesting measurements are really tests of self-consistency and of our understanding of QCD, rather than determinations of this parameter.

An example is the use of power corrections for the non-perturbative corrections to event shape variables, as described in Bryan Webber's contribution to these proceedings. I would place DELPHI's extraction of $\alpha_{s}$ from oriented event shape variables 47 in the same category. Fits at $m_{Z}$ yield very precise values of $\alpha_{s}: 0.1180 \pm 0.0018$ from 

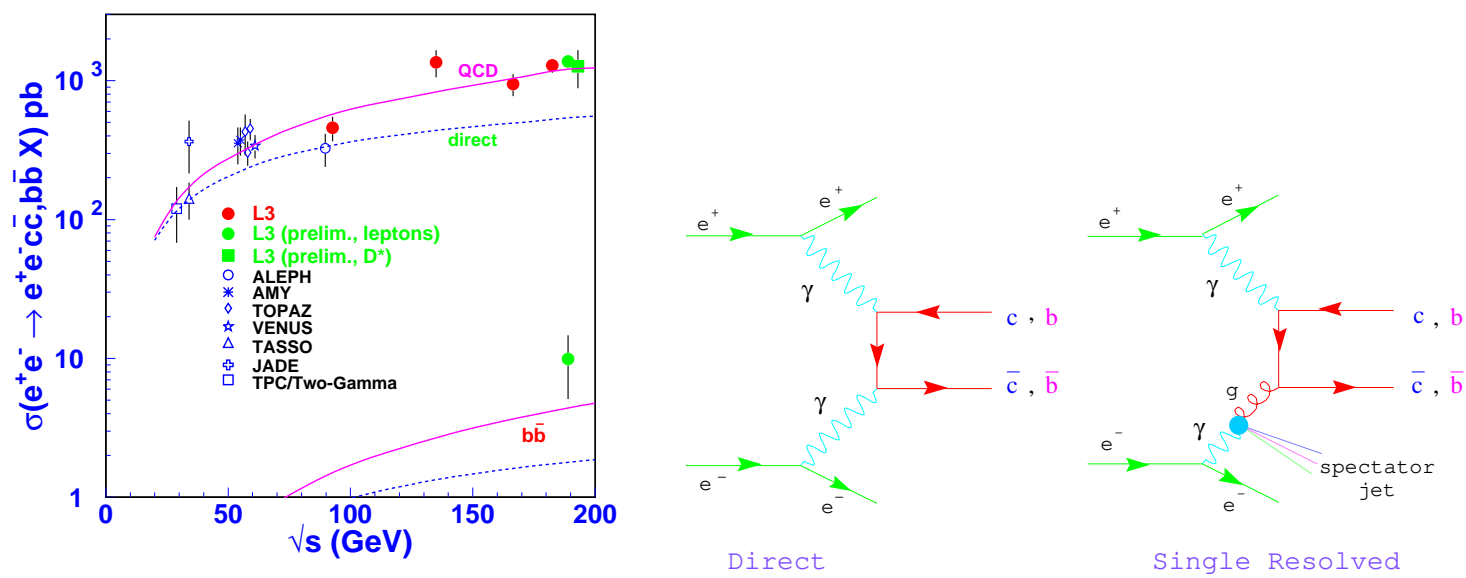

Figure 20: Cross sections for heavy quark production measured at LEP [30]. The Feynman diagrams show the direct and resolved photon contributions to the process.

the jet cone energy fraction, for example. However, the fitting procedure relies on "optimization" of the renormalization scale for each variable through a simultaneous fit of $\alpha_{s}$ and $x_{\mu}=\mu^{2} / Q^{2}$. For 18 jet shape variables the resulting scales range from $\mu^{2}=(0.003 Q)^{2}$ to $(7 Q)^{2}$, a much larger range than one is comfortable with. In fact the whole procedure has been called theoretically unjustified. Nonetheless the consistency of the results is certainly interesting: if $x_{\mu}$ is fixed to 1 , the spread in the extracted $\alpha_{s}$ values is much larger. I suspect that this is telling us something about QCD (though maybe not, or not just, the value of $\alpha_{s}$ ).

Another measurement of this type is the extraction of $\alpha_{s}$ from the CDF inclusive jet cross section 48]. The value quoted, $\alpha_{s}\left(m_{Z}\right)=0.113_{-0.009}^{0.008}$, is consistent with the world average, and $\alpha_{s}$ shows a nice evolution with scale (given by the jet transverse energy), as shown in Fig. 23. However the figure also shows that the measurement suffers from a large, and hard to quantify, sensitivity on the parton distributions, especially on the value of $\alpha_{s}$ assumed therein. At this time I think it must be characterized as a nice test of QCD and not a measurement of $\alpha_{s}$.

\section{Some Final Remarks}

In closing I will take the opportunity to outline some areas where I think progress would be welcome: "What I would like for Christmas".

Firstly parton distributions with quoted uncertainties, or at least with a technique 

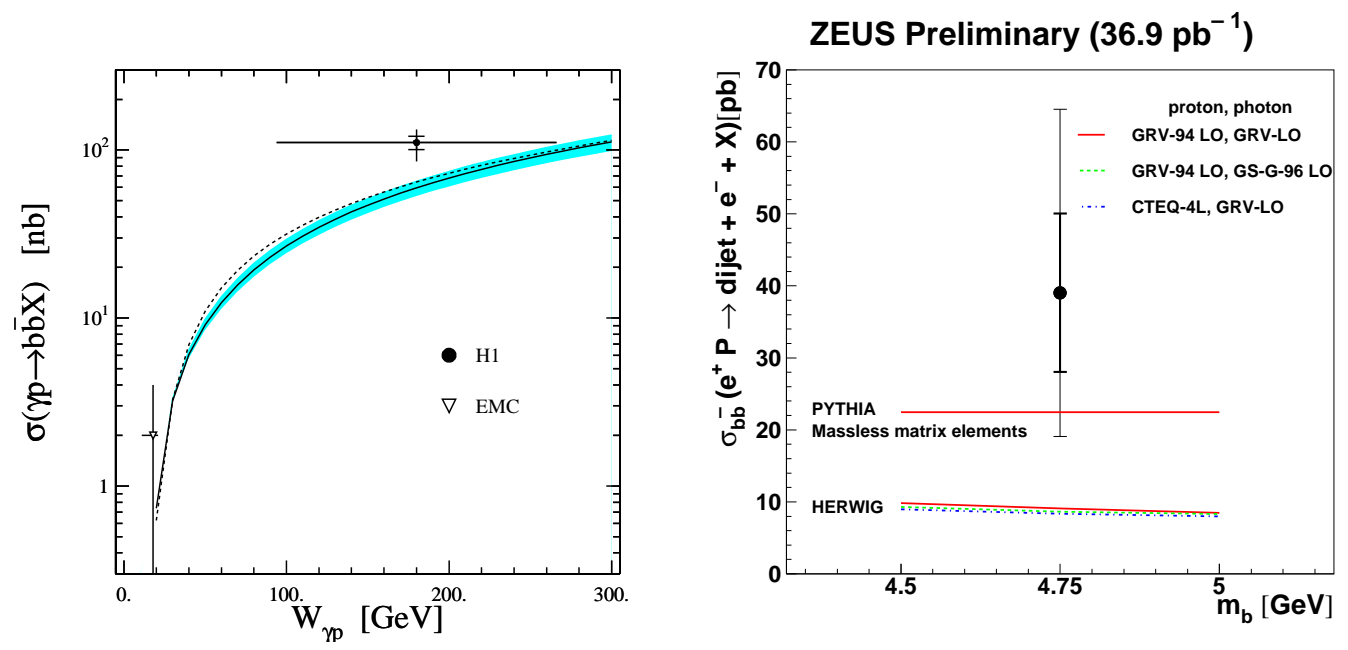

Figure 21: H1[31] and ZEUS[32] measurements of the $b$ production cross section at HERA, compared to NLO and LO QCD respectively.

for the propagation of uncertainties as outlined by Giele and collaborators. This would spare us from future unnecessary excitement over things like the high- $E_{T}$ jet "excess."

Secondly I would like to see a theoretical and experimental effort to understand the underlying event in hadronic collisions. It is an inconsistent treatment of the event to subtract it out of the jet energies, as is usually done. The ratio of 1800 to $630 \mathrm{GeV}$ jet cross sections may indicate problems with this approach. And such understanding would also enable a consistent treatment of double parton scattering which may be very important at the LHC.

Thirdly we need progress on jet algorithms for hadron colliders. Indeed, there is a lot of work going on in workshops at Fermilab and Les Houches. There are various species of $k_{T}$ algorithm to be compared, and the question remains whether the cone algorithm can be made theoretically acceptable. Theoertical requirements centre on the need for infrared and collinear safety, and the avoidance of ad hoc parameters; experimentalists worry about sensitivity to noise, pileup and negative energies. These can have potentially large effects on jet measurements especially at low energies, as shown in Fig. 24.

Fourthly there is one QCD process that we have completely failed to describe so far. Figure 25 shows a CDF event with a track in the Roman Pot detectors. It is jet production, a perturbative process which I have claimed is well-modelled by NLO QCD. Except for one detail: in a substantial fraction (a few percent?) of such events, one of the protons doesn't break up. Whether we call this pomeron exchange, or 


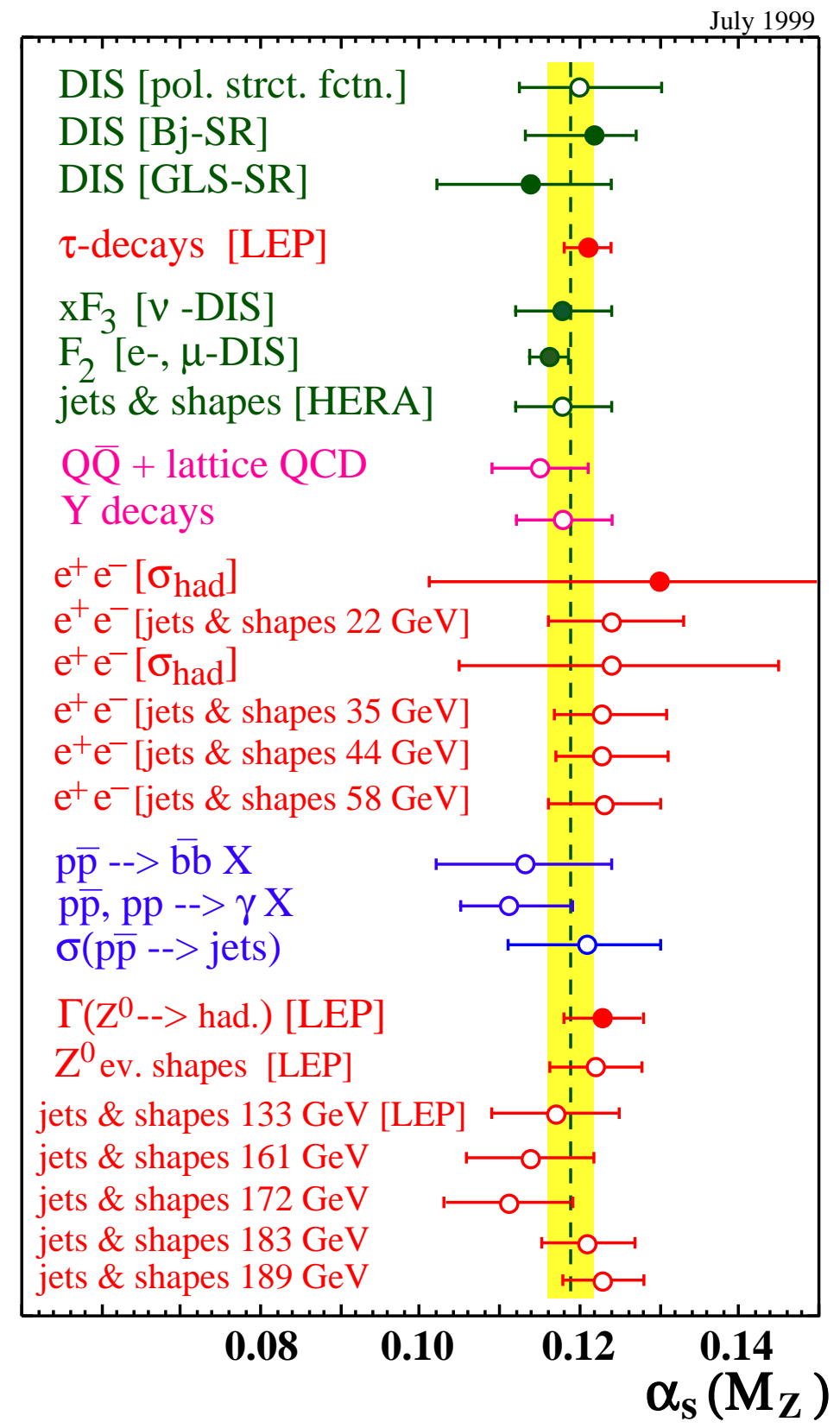

Figure 22: Compilation of $\alpha_{s}\left(m_{Z}\right)$ measurements. The summer 1999 world average value is $0.119 \pm 0.003$ [45]. 


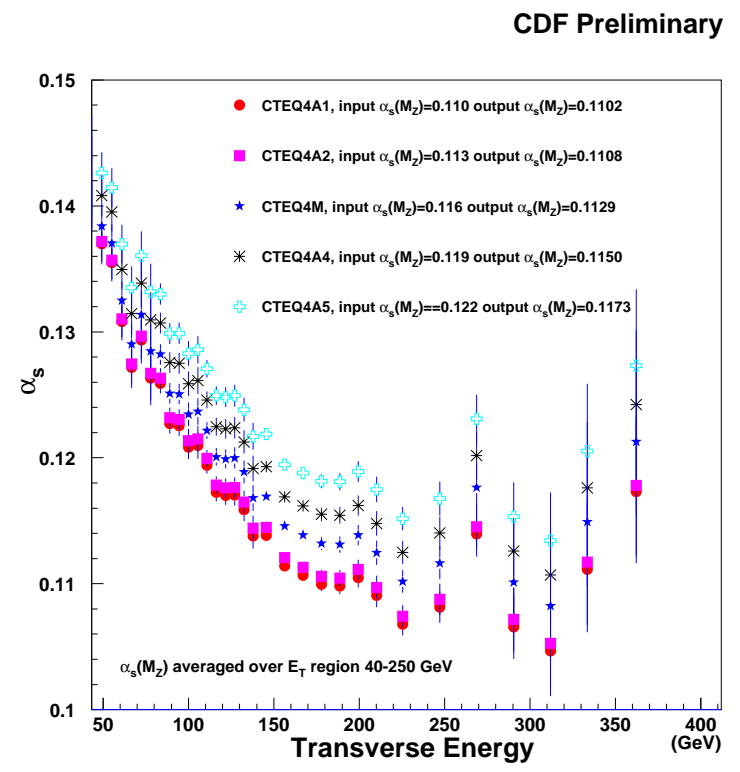

Figure 23: Value of $\alpha_{s}$ as a function of scale (jet transverse energy) inferred by CDF from the jet cross section using the CTEQ4A series of parton distributions [48].

think of it in terms of parton correlations inside the proton, it doesn't form part of a consistent description of hard hadronic interactions. My hunch is that we won't get too far in understanding processes like this as long as we think of them as being somehow alien to perturbative QCD.

\section{Conclusions}

In conclusion, testing QCD typically means testing our ability to calculate within QCD. In fact our calculational tools are working quite well, especially at moderate to high energy scales. The state of the art is NNLO calculations, and NLL resummations. However, interesting things (challenges!) start happening as we reduce the energy scale to of order $5 \mathrm{GeV}$. We have problems calculating $b$ quark cross sections; there are problems with low- $p_{T}$ direct photon production $\left(k_{T}\right.$ ?); and perhaps indications of effects of a few $\mathrm{GeV}$ in jet energies. In addition, there are other challenges for the future: identification of appropriate jet algorithms, understanding of the underlying event in hadron-hadron collisions, understanding parton distribution uncertainties, and obtaining a consistent picture of hard diffraction processes. With two new hadron collider facilities coming on line in the next decade, we can be assured of a vibrant future for perturbative QCD and jet physics. 


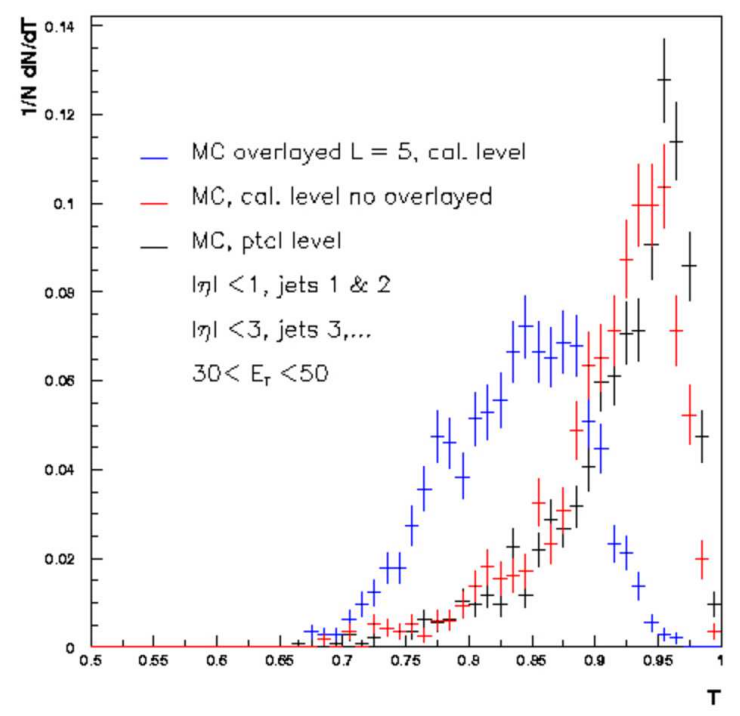

Figure 24: The importance of noise to jet algorithms. The figure shows a DO simulation of thrust distributions evaluated using $k_{T}$ jets. The calorimeter measures a distribution close to the ideal, until noise is added to the simulation, at which point it is dramatically shifted to lower values.

\section{References}

[1] F. Abe et al. [CDF Collaboration], Phys. Rev. Lett. 77, 438 (1996).

[2] B. Abbott et al. [D0 Collaboration], Phys. Rev. Lett. 82, 2451 (1999).

[3] G.C. Blazey and B.L. Flaugher, hep-ex/9903058.

[4] Presented by L. Babukhadia (for the DO Collaboration) at the XXIX Symposium on Multiparticle Dynamics (ISMD99), Brown University, Providence, RI, August 1999.

[5] CDF Collaboration, presented at the 13th Topical Conference on Hadron Collider Physics, Tata Institute of Fundamental Research, Mumbai, India, January 1999.

[6] C. Mesropian, (for the CDF Collaboration) Fermilab-Conf-99/177-E, in the Proceedings of the 34th Rencontres de Moriond: QCD and Hadronic Interactions, Les Arcs, France, March 1999. 

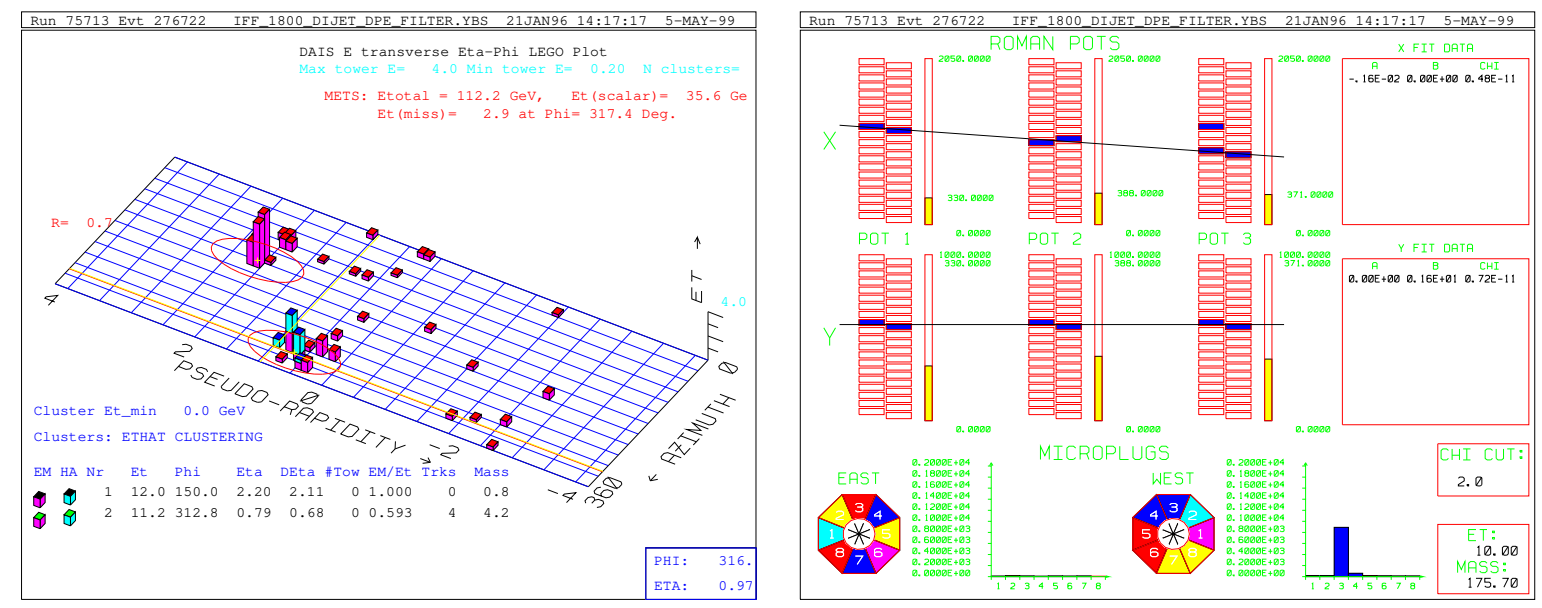

Figure 25: CDF double pomeron exchange dijet candidate. The left hand plot shows jets observed in the calorimeter; the right hand plot shows the two tracks in the roman pot detectors which are attributed to the undissociated proton and antiproton.

[7] J. Krane (for the CDF and DO Collaborations), in the Proceedings of the American Physical Society (APS) Meeting of the Division of Particles and Fields, University of California at Los Angeles, Los Angeles, CA, January 1999.

[8] Presented by A. Goussiou, (for the DO Collaboration), at the International Europhysics Conference on High-Energy Physics (EPS-HEP 99), Tampere, Finland, July 1999.

[9] R. Snihur (for the DO Collaboration), Fermilab-Conf-99/208-E, hep-ex/9907059, contributed paper to the International Europhysics Conference on High-Energy Physics (EPS-HEP 99), Tampere, Finland, July 1999.

[10] See for example the presentations of J. Terron (ZEUS) and C. Glasman (H1) at the International Europhysics Conference on High-Energy Physics (EPS-HEP 99), Tampere, Finland, July 1999.

[11] ZEUS collaboration, contributed paper to the International Europhysics Conference on High-Energy Physics (EPS-HEP 99), Tampere, Finland, July 1999.

[12] F. Abe et al. [CDF Collaboration], Phys. Rev. Lett. 73, 2662 (1994).

[13] S. Abachi et al. [D0 Collaboration], Phys. Rev. Lett. 77, 5011 (1996) hepex/9603006. 
[14] Presented by S. Kuhlmann (for the CDF Collaboration) at the 7th International Workshop on Deep Inelastic Scattering and QCD (DIS 99), Zeuthen, Germany, April 1999.

[15] H. Baer, J. Ohnemus and J.F. Owens, Phys. Rev. D42, 61 (1990).

[16] P. Hanlet [D0 Collaboration], Nucl. Phys. Proc. Suppl. 64, 78 (1998).

[17] W. Vogelsang, private communication.

[18] L. Apanasevich et al. [Fermilab E706 Collaboration], Phys. Rev. Lett. 81, 2642 (1998).

[19] P. Aurenche, M. Fontannaz, J.P. Guillet, B. Kniehl, E. Pilon and M. Werlen, Eur. Phys. J. C9, 107 (1999).

[20] S. Catani, M.L. Mangano, P. Nason, C. Oleari and W. Vogelsang, JHEP 03, 025 (1999).

[21] N. Kidonakis, hep-ph/9905480.

[22] Presented by P. Saull (for the ZEUS Collaboration), at the International Europhysics Conference on High-Energy Physics (EPS-HEP 99), Tampere, Finland, July 1999.

[23] B. Abbott et al. [D0 Collaboration], hep-ex/9909020.

[24] G.A. Ladinsky and C.P. Yuan, Phys. Rev. D50, 4239 (1994).

[25] C.T. Davies, B.R. Webber and W.J. Stirling, Nucl. Phys. B256, 413 (1985).

[26] Ellis and Veseli R.K. Ellis and S. Veseli, Nucl. Phys. B511, 649 (1998).

[27] J.R. Dittmann, UMI-99-17363. These results are available at http://www-cdf.fnal.gov.

[28] F. Abe et al. [CDF Collaboration], Phys. Rev. Lett. 71, 2396 (1993); Phys. Rev. D53, 1051 (1996).

[29] S. Abachi et al. [D0 Collaboration], Phys. Rev. Lett. 74, 3548 (1995); B. Abbott et al. [D0 Collaboration], hep-ex/9905024.

[30] L3 note 2427, unpublished.

[31] C. Adloff et al. [H1 Collaboration], Phys. Lett.B 467, 156 (1999). 
[32] Presented by C.L. Kim (for ZEUS collaboration) at the International Europhysics Conference on High-Energy Physics (EPS-HEP 99), Tampere, Finland, July 1999.

[33] F.Abe et al. [CDF collaboratipn], Phys. Rev. Lett. 82, 2808(E) (1999).

[34] B. Abbott et al. [D0 Collaboration], Phys. Rev. D60, 012001 (1999).

[35] R. Bonciani, S. Catani, M.L. Mangano and P. Nason, Nucl. Phys. B529, 424 (1998).

[36] E.L. Berger and H. Contopanagos, Phys. Rev. D57, 253 (1998).

[37] N. Kidonakis, hep-ph/9904507.

[38] J. Santiago and F.J. Ynduráin, hep-ph/9904344.

[39] A.L. Kataev, G. Parente and A.V. Sidorov, hep-ph/9905310.

[40] Presented by G. Quast (for the LEP Electroweak Working Group), at the International Europhysics Conference on High-Energy Physics (EPS-HEP 99), Tampere, Finland, July 1999.

[41] L3 note 2440, unpublished.

[42] OPAL note PN-415, unpublished.

[43] H1 collaboration, contributed paper to the International Europhysics Conference on High-Energy Physics (EPS-HEP 99), Tampere, Finland, July 1999

[44] ZEUS collaboration, contributed paper to the International Europhysics Conference on High-Energy Physics (EPS-HEP 99), Tampere, Finland, July 1999

[45] S. Bethke, private communication.

[46] S. Bethke, hep-ex/9812026.

[47] DELPHI note 99-122, unpublished.

[48] CDF note 4892, unpublished. These results are available at http://wwW-cdf.fnal.gov. 


\section{Discussion}

Alex Firestone (NSF): Regarding the isolated inclusive photon distributions, how plausible is it that at least some of the problem is due to signal extraction above background? Different experiments have different calorimeter resolution and different criteria for photon isolation. Could this be the source of the inconsistencies?

Womersley: Having carried out these analyses myself at the Tevatron, I certianly understand that there are large systematic errors at low $E_{T}$ because of the big jet backgrounds. There are also problems in computing the effects of the isolation requirements on the theoretical predictions. But the difficulty pointed out by Aurenche et al. is that the E706 and the ISR data do not agree. I do not really want to speculate on why that might be so.

Jonathan Butterworth (University College London): It's true that the photon structure function is a way to resum partonic contributions. But the partons from the photon are collinear, so the $k_{T}$ effect is not included in this calculation.

Paul Söding (DESY): One of your wishes for Christmas has already become true; there is an analysis by Michael Botje which provides PDF's with uncertainties. The paper is available.

Michel Davier (LAL, Orsay): I think that the $\alpha_{s}$ average you quoted from Siggi Bethke has a very conservative uncertainty (0.003). The 2 most precise determinations (from $\tau$ decays and the $Z$ width) have uncertainties already at this level and the jet rate at LEP yields results approaching this accuracy. These determinations have different systematics. So I would rather think that the combined uncertainty is 0.002 at most.

Siggi Bethke (Aachen and MPI Munich): Most of the individual errors quoted are lower limits, because there is some freedom to adjust the theoretical uncertainties. The new world average that was quoted relies, for the first time, on NNLO calculations alone, and also assumes some degree of correlation between individual errors. The scatter of the central values of the results is also \pm 0.003 . 